\title{
Effects of Behavioral Tactics of Predators on Dynamics of a Predator-Prey System
}

\author{
Hui Zhang, ${ }^{1}$ Zhihui Ma, ${ }^{2}$ Gongnan Xie, ${ }^{3}$ and Lukun Jia ${ }^{4}$ \\ ${ }^{1}$ Department of Applied Mathematics, School of Natural and Applied Sciences, Northwestern Polytechnical University, Xi'an, \\ Shaanxi 710027, China \\ ${ }^{2}$ Institute of Bioinformatics, School of Mathematics and Statistics, Lanzhou University, Lanzhou 730000, China \\ ${ }^{3}$ School of Mechanical Engineering, Northwestern Polytechnical University, Xian, Shaanxi 710027, China \\ ${ }^{4}$ School of Mathematical Sciences, Xiamen University, Xiamen 361005, China
}

Correspondence should be addressed to Hui Zhang; huizhang@nwpu.edu.cn

Received 8 April 2014; Accepted 11 May 2014; Published 26 May 2014

Academic Editor: Xiao-Jun Yang

Copyright (C) 2014 Hui Zhang et al. This is an open access article distributed under the Creative Commons Attribution License, which permits unrestricted use, distribution, and reproduction in any medium, provided the original work is properly cited.

A predator-prey model incorporating individual behavior is presented, where the predator-prey interaction is described by a classical Lotka-Volterra model with self-limiting prey; predators can use the behavioral tactics of rock-paper-scissors to dispute a prey when they meet. The predator behavioral change is described by replicator equations, a game dynamic model at the fast time scale, whereas predator-prey interactions are assumed acting at a relatively slow time scale. Aggregation approach is applied to combine the two time scales into a single one. The analytical results show that predators have an equal probability to adopt three strategies at the stable state of the predator-prey interaction system. The diversification tactics taking by predator population benefits the survival of the predator population itself, more importantly, it also maintains the stability of the predator-prey system. Explicitly, immediate contest behavior of predators can promote density of the predator population and keep the preys at a lower density. However, a large cost of fighting will cause not only the density of predators to be lower but also preys to be higher, which may even lead to extinction of the predator populations.

\section{Introduction}

The investigation of phenomena involving complex geometry, patterns, and scaling has undergone a substantial development in the past decades. In natural reality, many objects are "self-similar" at different scales, which is the essence of fractal. We see fractal patterns almost everywhere in nature, even the more random-appearing patterns of clouds or coastlines. Fractal has been applied into a variety of areas as a new concept and method, scaling is another important concept with it. In ecosystem, an ecological process including some small parts which happen on different scales is commonly observed; it is possible to take advantage of these time scales in order to reduce the dimension of the initial complete model and to build a simplified system that describes the dynamics of a small number of global variables. Aggregation methods are extended to systems with different time scales to reduce the number of variables [1-6].
Game theory as a very useful branch of mathematics has long been widely used to investigate the interaction between rational players in a game, the core problem in game theory is what kind of strategies will be adopted to improve player's payoff as much as possible. Smith and Price [7] ingeniously linked its concept of payoff functions and players to the biological terminology of fitness and individuals, respectively, in order to explain the evolution of ritualised conflicts within species, for example, when individuals compete for mates or territory. Their seminal work thus inspired an entirely new approach to behavior ecology; evolutionary game theory is born to investigate problems involving frequency-dependent selection. It deals with entire populations of individuals, all programmed to use some strategies (or types of behavior); strategies with higher payoff will spread within population, through learning, copying, or inheriting, even by infection. The payoffs depend on the actions of coplayers and hence on the frequencies of the strategies within the populations. 
In population biology, understanding the effects of interacting species at the population and community level is one of the most important issues. The classical predator-prey system is considered as a basic aspect and has long been investigated since Lotka in 1920 [8] followed by Volterra [9] from a theoretical point of view, respectively, where a simple coupled set of differential equations is devised to describe an autocatalytic reaction and the statistics of fish catches in the Adriatic. This first model (to be called Lotka-Volterra model (LVM)) has since become one of the central paradigms to elucidate the main features of species interactions and population cycles in predator-prey systems, where predator depends on the prey for food, and each species has an inherent birth and death rate. The two populations end up oscillating, with the predator population crests slightly offset from that of prey. Based on LVM, a proliferation of models has been employed to qualitatively capture and qualitatively understand the primary features of predator-prey systems as well as competition system (e.g., [10-12]), by considering factors which influence the stable coexistence of ecosystem [13] and maintenance of biodiversity [14-17].

Individual's behaviors play an important role in population dynamics $[18,19]$. Individuals have the ability of learning and can change tactics along their life according to the environment conditions and the results of past contests. Behavioral plasticity [20] allows an individual to be more flexible to adopt the behavior that can optimize its survival in the present environment [21]. Typically, aggressive behavior is commonly observed in animal kingdom when the prey resource is rare, thus making predators food-deprived to monopolize the limited resources, for example, in vertebrates [22], in birds [23], and in mammals [24]. Particularly, in a real world, predators are expected to choose their strategy both in relation to the density of prey and in relation with the strategies adopted by the other competitors. Inspired by this point, Auger et al. [6] studied a predator-prey system incorporating predators using the classical hawk and dove tactics. In their work, individual behavior structure of predators is firstly incorporated into the classical predatorprey model.

In this paper, a traditionally defined Lotka-Volterra interaction system is used to investigate the dynamics of predatorprey system, where predators use different strategies when disputing a prey. The canonical rock-paper-scissors (R-PS) strategy [17] is assumed, with the second out-competing the first, the third out-competing the second, and the first out-competing the third, which has been invoked to capture the remarkable coexistence of three types of cyclically competition. The principle aim of this study is to explore the effect of individual behavior on the dynamics of the predatorprey system. In particular, we will look at the existence of coexistence equilibria between the prey and predator and the corresponding internal structure of the predator population: monomorphic or polymorphic state. We are interested in the coevolution at the individual and community level.

At first, we present the game dynamic model representing the fast scale part of the complete system in Section 2.1. Then the slow scale part is built up based on the classical LVM with prey-density dependence. Combining the fast and slow scale parts together, Sections 2.3 and 2.4 are devoted to the construction and analysis of the ensuing aggregated model. The dynamics of the system can be studied by mathematical analyses and computer simulations, some details of the analysis are given in the appendix.

\section{Model and Analysis}

Suppose that all predators hunt the same prey, after a predator catches a prey, this predator has to fight with others to keep the prey. Simplifying, we assume that once a prey is killed, a second predator in the vicinity arrives and is disputing the prey to the predator that has caught the prey. Predators can use three behavior tactics rock, paper, and scissors to dispute a prey when they meet. After a certain number of encounters, on average, the tactic that gives predators the better payoff will be carried out. This dynamic process can be described by replicator equations [25-30].

Because predators encounter and fight frequently (for example, each day) to keep a captured prey or to dispute a prey to another predator, we suppose the game dynamics correspond to a fast time scale. However, a few preys are killed each day in comparison to the total reservoir of prey, so the predator-prey interaction terms correspond to a slow time scale, involving birth, death, and predation.

Denote $n(t)$ and $p(t)$ as the densities of prey and predator population at time $t$, respectively. Next, we will construct models to describe the interaction of predators and prey.

2.1. Replicator Dynamics of Predators on a Fast Time Scale. Let us denote $a_{i j}(i, j \in\{1,2,3\}, 1,2,3$ represent rock, paper, scissors, respectively) as the element of payoff matrix $A$, which corresponds to the gain that is obtained by an individual playing tactic $i$ against an individual playing tactic $j$. When two individuals with the same behavior meet, they share the gain $G$ equally but also the cost $C(C>0)$ due to fighting, $a_{i i}=(G-C) / 2$, and vice versa, the winner gets $G$ while the loser gets nothing. For example, when a rock strategy individual meets a paper strategy one, the first one gets nothing, but another gets $G$. Consequently, the payoff matrix $A$ takes the following form:

$$
\mathbf{A}=\left(\begin{array}{ccc}
\frac{G-C}{2} & 0 & G \\
G & \frac{G-C}{2} & 0 \\
0 & G & \frac{G-C}{2}
\end{array}\right)
$$

The gain $G$ of the game corresponds to the prey amount that two predators dispute each day. To simplify, a classical type I linear functional response is taken into account, that is,

$$
G=a n,
$$

where $a$ is a positive parameter.

Denote $p_{r}(t), p_{p}(t)$, and $p_{s}(t)$ as the densities of individuals with rock, paper, and scissors strategies at time $t$, respectively. The total density of predators will be

$$
p(t)=p_{r}(t)+p_{p}(t)+p_{s}(t) .
$$


Furthermore, let $x_{1}(t), x_{2}(t)$, and $x_{3}(t)$ be respectively the rock, paper, and scissor proportions in the population of predators at time $t$ as follows:

$$
\begin{gathered}
x_{1}(t)=\frac{p_{r}(t)}{p(t)}, \quad x_{2}(t)=\frac{p_{p}(t)}{p(t)}, \\
x_{3}(t)=1-x_{1}(t)-x_{2}(t) .
\end{gathered}
$$

The replicator equations describing the evolutionary dynamics of the three strategies read as follows:

$$
\begin{gathered}
\frac{\mathrm{d} x_{i}}{\mathrm{~d} \tau}=x_{i}\left[(\mathbf{A} \mathbf{x})_{i}-\mathbf{x}^{T} \mathbf{A} \mathbf{x}\right], \quad i=1,2,3, \\
\mathbf{x}^{T} \mathbf{A} \mathbf{x}=\sum_{j=1}^{3} x_{j}(\mathbf{A x})_{j},
\end{gathered}
$$

where $\mathbf{x}=\left(x_{1}, x_{2}, x_{3}\right)$. Obviously, if a strategy brings a better payoff than the average payoff of the population, the proportion of individuals playing this strategy is increasing and conversely is decreasing. In (5), $\tau$ is the fast time scale. Thus, we assume that the R-P-S game is in fast time scale compared to the processes that we will now consider in the model, such as predator death, prey growth, and capturing of preys by predators.

2.2. Dynamics of Predator-Prey at the Slow Time Scale. A classical Lotka-Volterra model with self-limiting prey [31] is assumed to describe the total prey density as follows:

$$
\frac{\mathrm{d} n}{\mathrm{~d} t}=r n\left(1-\frac{n}{K}\right)-a n p,
$$

where $t$ corresponds to the slow time scale. Usually, we have the relationship $t=\epsilon \tau$ for two time scales. From (6) we know, in absence of predators, that the growth of prey population depends on a logistic equation with an intrinsic growth rate $r$ towards a carrying capacity $K$. Besides, we assume a LotkaVolterra functional response of type $I$; the parameter $a$ is positive, which represents predation force and is the same as the one in the functional response.

Predators are assumed to have a constant natural mortality rate $\mu(\mu>0)$ no matter what kind of strategies they use., the growth rate of each subgroup with the same strategy is proportional to the average payoff obtained by an individual using the tactic on the occasion of each type of encounter. For example, a rock player can encounter either a rock player in proportion of $p_{r} / p$ and gets the gain $(G-C) / 2$, or a paper player in proportion of $p_{p} / p$ and gets zero, or as a proportion of $p_{s} / p$ to encounter a scissor player and gets $G$. Consequently, the growth of the rock predator subpopulation is described as follows:

$$
\frac{\mathrm{d} p_{r}}{\mathrm{~d} t}=-\mu p_{r}+p_{r}\left(\alpha \frac{G-C}{2} \frac{p_{r}}{p}+\alpha G \frac{p_{s}}{p}\right),
$$

where $\alpha$ is a conversion positive coefficient of gain and cost into biomass of predators. Analogously, it also holds for the paper subpopulation of predators as follows:

$$
\frac{\mathrm{d} p_{p}}{\mathrm{~d} t}=-\mu p_{p}+p_{p}\left(\alpha G \frac{p_{r}}{p}+\alpha \frac{G-C}{2} \frac{p_{p}}{p}\right) .
$$

For the scissors subpopulation of predators, we obtain

$$
\frac{\mathrm{d} p_{s}}{\mathrm{~d} t}=-\mu p_{s}+p_{s}\left(\alpha G \frac{p_{p}}{p}+\alpha \frac{G-C}{2} \frac{p_{s}}{p}\right) .
$$

2.3. The Complete Slow-Fast Predator-Prey Model. By combing both fast and slow processes in a similar way as in previous papers [6, 32-35], the complete model reads as follows:

$$
\begin{aligned}
& \epsilon \frac{\mathrm{d} n}{\mathrm{~d} t}=\epsilon\left(r n\left(1-\frac{n}{K}\right)-a n p\right) . \\
& \epsilon \frac{\mathrm{d} p_{r}}{\mathrm{~d} t}=\epsilon\left(-\mu p_{r}+p_{r}\left(\alpha \frac{G-C}{2} \frac{p_{r}}{p}+\alpha G \frac{p_{s}}{p}\right)\right) \\
&+p x_{1}\left((\mathbf{A x})_{1}-\mathbf{x}^{T} \mathbf{A} \mathbf{x}\right) . \\
& \epsilon \frac{\mathrm{d} p_{p}}{\mathrm{~d} t}=\epsilon\left(-\mu p_{p}+p_{p}\left(\alpha G \frac{p_{r}}{p}+\alpha \frac{G-C}{2} \frac{p_{p}}{p}\right)\right) \\
&+p x_{2}\left((\mathbf{A x})_{2}-\mathbf{x}^{T} \mathbf{A} \mathbf{x}\right) \cdot \\
& \epsilon \frac{\mathrm{d} p_{s}}{\mathrm{~d} t}= \epsilon\left(-\mu p_{s}+p_{s}\left(\alpha G \frac{p_{p}}{p}+\alpha \frac{G-C}{2} \frac{p_{s}}{p}\right)\right) \\
&+p x_{3}\left((\mathbf{A x})_{3}-\mathbf{x}^{T} \mathbf{A} \mathbf{x}\right),
\end{aligned}
$$

where $\epsilon \ll 1$ is a small parameter. By using the fast time scale $\tau$, the system changes to

$$
\begin{aligned}
& \frac{\mathrm{d} n}{\mathrm{~d} \tau}=\epsilon\left(r n\left(1-\frac{n}{K}\right)-a n p\right) . \\
\frac{\mathrm{d} p_{r}}{\mathrm{~d} \tau}= & \epsilon\left(-\mu p_{r}+p_{r}\left(\alpha \frac{G-C}{2} \frac{p_{r}}{p}+\alpha G \frac{p_{s}}{p}\right)\right) \\
& +p x_{1}\left((\mathbf{A x})_{1}-\mathbf{x}^{T} \mathbf{A} \mathbf{x}\right) . \\
\frac{\mathrm{d} p_{p}}{\mathrm{~d} \tau}=\epsilon & \left(-\mu p_{p}+p_{p}\left(\alpha G \frac{p_{r}}{p}+\alpha \frac{G-C}{2} \frac{p_{p}}{p}\right)\right) \\
& +p x_{2}\left((\mathbf{A x})_{2}-\mathbf{x}^{T} \mathbf{A} \mathbf{x}\right) . \\
\frac{\mathrm{d} p_{s}}{\mathrm{~d} \tau}= & \epsilon\left(-\mu p_{s}+p_{s}\left(\alpha G \frac{p_{p}}{p}+\alpha \frac{G-C}{2} \frac{p_{s}}{p}\right)\right) \\
& +p x_{3}\left((\mathbf{A x})_{3}-\mathbf{x}^{T} \mathbf{A} \mathbf{x}\right) .
\end{aligned}
$$

Obviously, the game dynamics correspond to the fast time scale while the small terms of the order of $\epsilon$ correspond to the slow time scale. Next, aggregation method will be carried to reduce the dimension of the system.

\subsection{The Aggregated Predator-Prey Model}

2.4.1. Derivation of the Aggregated Model. Since $\epsilon \ll 1$, we neglect the small terms of the order of $\epsilon$ and do stability 
analysis for the fast part of the system which relates to the game dynamics as follows:

$$
\begin{aligned}
& \frac{\mathrm{d} p_{r}}{\mathrm{~d} \tau}=p x_{1}\left((\mathbf{A x})_{1}-\mathbf{x}^{T} \mathbf{A} \mathbf{x}\right) \\
& \frac{\mathrm{d} p_{p}}{\mathrm{~d} \tau}=p x_{2}\left((\mathbf{A x})_{2}-\mathbf{x}^{T} \mathbf{A} \mathbf{x}\right) \\
& \frac{\mathrm{d} p_{s}}{\mathrm{~d} \tau}=p x_{3}\left((\mathbf{A x})_{3}-\mathbf{x}^{T} \mathbf{A} \mathbf{x}\right)
\end{aligned}
$$

Since we are only interested in positive solutions, by realizing the fact that $x_{1}+x_{2}+x_{3}=1$ always holds at any time $t$, we can limit our analysis on the region called a three-dimensional simplex, $S^{3}=\left\{\left(x_{1}, x_{2}, x_{3}\right) \in R_{+}^{3}: x_{1}+x_{2}+x_{3}=1\right\}$. By letting the right-hand side of (12) equal zero, the equilibria of the system can be derived, which depends on the parameters $G$ and $C$.

The system has an interior stable focus $(1 / 3,1 / 3,1 / 3)$ no matter what the parameters are. There exist three fixed points $(1,0,0),(0,1,0)$, and $(0,0,1)$ located on the vertex of $S^{3}$; they are all saddle points given $G>C$, unstable equilibrium points for the inverse condition of $G<C$. Besides, three boundary equilibria $((G+C) / 2 C, 0,(-G+C) / 2 C)),((-G+C) / 2 C),(G+$ $C) / 2 C, 0)$, and $(0,(-G+C) / 2 C),(G+C) / 2 C)$ can also exist as saddle points provided that $G<C$. Therefore, $x^{*}=$ $(1 / 3,1 / 3,1 / 3)$ is the only nontrivial interior asymptotically stable point without depending on initial conditions. In this case, the predator population is a polymorphic state with equal probability of three tactics of predators.

In order to aggregate, we make the assumption that the fast process is at fast equilibrium. Then we come back to the complete initial system (10), substitute the previous fast equilibrium and add the three predator equations together. It is necessary to replace the fast variables in terms of the fast equilibrium which are $p_{r}=p / 3, p_{p}=p / 3$, and $p_{s}=p / 3$.

After some algebra, one obtains the following system of two equations governing the total prey and predator densities at the slow time scale, that we call the aggregated model (AM) as follows:

$$
\begin{aligned}
& \frac{\mathrm{d} n}{\mathrm{~d} t}=r n\left(1-\frac{n}{K}\right)-a n p+o(\epsilon) . \\
& \frac{\mathrm{d} p}{\mathrm{~d} t}=-\mu p+\alpha \frac{3 G-C}{2} p+o(\epsilon) .
\end{aligned}
$$

Because $\epsilon$ is small enough and the system is structurally stable [36]; it is a good approximation of the real dynamics. Then by neglecting these terms of higher order of $\epsilon$ and realizing the fact that $G(n)=a n$, the aggregated model simply reads as follows:

$$
\begin{gathered}
\frac{\mathrm{d} n}{\mathrm{~d} t}=\left(r-r \frac{n}{K}-a p\right) n \\
\frac{\mathrm{d} p}{\mathrm{~d} t}=\left(-\mu+\frac{3 \alpha a n}{2}-\frac{\alpha C}{2}\right) p .
\end{gathered}
$$

2.4.2. Analyses of the Dynamics of the Aggregated Model. The phase plane $\{n, p\}$ is separated by the vertical nullcline $L$ denoted as $r K-r n-a p K=0$ and the horizontal nullcline $M$ denoted as $-2 \mu+3 \alpha a n-\alpha C=0$ into different regions, both the signs of $\mathrm{d} n / \mathrm{d} t$ and $\mathrm{d} p / \mathrm{d} t$ do not change in every part. Next, the dynamics of the system will be studied through the analysis of the intersection of the lines $L$ and $M$.

(1) $K<(2 u+\alpha C) /(3 \alpha a), L$ and $M$ are not intersected in phase plane.

In this case, the system has two equilibria, where $(0,0)$ is a saddle point while $(K, 0)$ is an asymptotically stable point (see Appendix A.1). There does not exist any limit cycle. We know that closed trajectory should enclose equilibrium point, but there is no equilibrium point in the interior part of the phase plane. Therefore, the trajectories that originated from the vertical axis representing $n(t)=0$ will approach to the point $(0,0)$; otherwise, all trajectories will end at the point $(K, 0)$. That is to say, the dynamic result will die out of predators no matter what the initial densities of prey and predators are. Once the initial density of prey is not zero, it will reach to a stable density with a value of $K$ in the end.

(2) $K>(2 u+\alpha C) /(3 \alpha a), L$ and $M$ intersect in the phase plane.

The system includes three equilibria, $(0,0)$ and $(K, 0)$ are saddle points, the interior point $\bar{x}=(\bar{n}, \bar{p})=((2 u+$ $\left.\alpha C) /(3 \alpha a), r(3 \alpha a K-2 u-\alpha C) /\left(3 \alpha a^{2} K\right)\right)$ standing for the intersection point of $L$ and $M$ is an asymptotically stable point (see Appendix A.1). In addition, we can testify that there is no limit cycle, the details are written in Appendix A.3. Therefore, once the initial densities of prey and predators are all larger than zero, their density will finally reach the point $\bar{x}$.

In reality, $\sigma=(2 u+\alpha C) /(3 \alpha a)$ is the least food requirement for viability of predators, and $K$ is the carrying capacity. $\sigma>K$ means that the least food requirement for predators is larger than the reproduction and fecundity of prey, so predators will extinct without any doubt, or else, the predators and preys will coexist together.

\section{Results}

A classical LVM will be studied in this part in order to give a comparison with our AM. Suppose that there is no difference between the strategy adopted by predators, the predator-prey interaction can be described as follows:

$$
\begin{gathered}
\frac{\mathrm{d} n}{\mathrm{~d} t}=r n\left(1-\frac{n}{K}\right)-a n p \\
\frac{\mathrm{d} p}{\mathrm{~d} t}=-\mu p+\alpha a n p .
\end{gathered}
$$

By using a similar method as in the last model, the dynamics can also be recognized to be two cases. When $K<u /(\alpha a)$, the system has two equilibria, $(0,0)$ is a saddle point, $(K, 0)$ is an asymptotically stable point. While for $K>u /(\alpha a)$, the system has three equilibria, both $(0,0)$ and $(K, 0)$ are saddle points, $\tilde{x}=(\tilde{n}, \tilde{p})=\left(u /(\alpha a), r(\alpha a K-u) /\left(\alpha a^{2} K\right)\right)$ 
TABLE 1: The evolutionary stable state in LVM and AM.

\begin{tabular}{|c|c|c|c|c|c|}
\hline$C<\frac{u}{\alpha}\left(\frac{2 u+\alpha C}{3 \alpha a}<\frac{u}{\alpha a}\right)$ & LVM & $\mathrm{AM}$ & $C>\frac{u}{\alpha}\left(\frac{u}{\alpha a}<\frac{2 u+\alpha C}{3 \alpha a}\right)$ & LVM & $\mathrm{AM}$ \\
\hline$K<\frac{2 u+\alpha C}{3 \alpha a}$ & $(K, 0)$ & $(K, 0)$ & $K<\frac{u}{\alpha a}$ & $(K, 0)$ & $(K, 0)$ \\
\hline$\frac{2 u+\alpha C}{3 \alpha a}<K<\frac{u}{\alpha a}$ & $(K, 0)$ & $(\bar{n}, \bar{p})$ & $\frac{u}{\alpha a}<K<\frac{2 u+\alpha C}{3 \alpha a}$ & $(\widetilde{n}, \widetilde{p})$ & $(K, 0)$ \\
\hline \multirow[t]{2}{*}{$K>\frac{u}{\alpha a}$} & $(\widetilde{n}, \widetilde{p})$ & $(\bar{n}, \bar{p})$ & $K>\frac{2 u+\alpha C}{3 \alpha a}$ & $(\widetilde{n}, \widetilde{p})$ & $(\bar{n}, \bar{p})$ \\
\hline & $\tilde{n}>\bar{n}$ & $\widetilde{p}<\bar{p}$ & & $\tilde{n}<\bar{n}$ & $\tilde{p}>\bar{p}$ \\
\hline
\end{tabular}

A comparison between evolutionary stable state of the models LVM and AM is given. Parameters $C, K, u, \alpha, a$ represent fighting cost, carrying capacity of prey, natural mortality rate of prey, a conversion positive coefficient into biomass of predators, a positive parameter in functional response, respectively. Two cases are recognized by considering whether $C$ is smaller than $u /(\alpha a)$ or not. Furthermore, parameter region $\{K, u, \alpha, a, C\}$ can be divided into three parts according to the evolutionary stable state in two models for each case. It shows that the stable state for two modes appears as either kept a constant with only prey at carrying capacity, or coexistence of prey and predators with a variation of population density, or a change between only prey at carrying capacity and coexistence of prey and predators. The values of $(\widetilde{n}, \widetilde{p})$ and $(\bar{n}, \bar{p})$ are given in the main text.

is an asymptotically stable interior point. The details are given in Appendix A.2. In addition, we can verify there is no limit cycle in the phase plane by using the same method as in Appendix A.3. In summary, all results are concluded in Table 1.

Therefore, we find that the stable state of the predatorprey system will be changed somehow considering that predator population uses different strategies when disputing a prey, which can be recognized as four cases depending on different parameter settings. The stable state can change from only preys with a value at carrying capacity in LVM to coexistence between preys and predators in AM, Figures 1(a) and 1(b) give an example. It also shows from the coexistence of prey and predators in LVM to only prey with a value at carrying capacity in AM (see Figures 2(a) and 2(b)). In addition, the case that the prey population with the density of carrying capacity but predators dying out can be held. Coexistence of the predator and prey populations but with a variation of population densities in the two models can also be kept. When $C<u / \alpha$, for the parameter region $(2 u+$ $\alpha C) /(3 \alpha a)<K<u /(\alpha a)$, the density of prey in the LVM will be higher than that in the AM at the stable state, but the result is inverse for the predators, as shown in Figures 1(c) and 1(d); while for $C>u / \alpha$, when the parameters satisfy $u /(\alpha a)<K<$ $(2 u+\alpha C) /(3 \alpha a)$, the density of prey in the LVM will be lower than that in the AM, but it is inverse for the predator populations, Figures 2(c) and 2(d) are an example. In conclusion, we can note that fighting between predators could promote the growth of the predator population when the coming cost is smaller than the division of natural mortality of prey by conversion coefficient, so a moderate degree of fighting will also benefit the evolution of the predator population. In other words, a slight extent of competition is helpful for the survival of the population. On the other side, fighting will naturally cause a negative effect on the survival of predator population when the cost is relatively large, thus making an acceleration of the prey population, this phenomenon confirms the reality.

\section{Conclusions and Discussion}

A vulnerable subject in population ecology is understanding that how diversity and complexity of natural populations contribute to the overall stability and persistence of ecological systems. The idea that diversity promotes stability is intuitively appealing. In this work, the stability of a predatorprey interaction system with predators using diversification strategies is investigated to study the effect of different tactics taken by predators when disputing a prey, thus providing us a better understanding of the important effect of biodiversity on the stability of the ecosystem.

In this study, a dynamic modeling method [37] is employed to explore the dynamics of a predator-prey system by connecting individual behavior with population dynamics. Particularly, by resorting to game theory, replicator equations are constructed to derive ESS of the fast dynamics describing the variation of frequencies of different tactics. Here, by taking the R-P-S strategy to investigate the effect of predator behavior on the stability of predator-prey system, we found that the ESS in fast time scale of disputing a prey is independent of parameters and is very symmetric, which locates on the center of the strategy space. This result is dramatically different from that by Auger et al. [6], where the ESS in the fast time scale will stay on different equilibrium points with a variation of the density of preys in their system. Therefore, the main conclusion of their studies is that there exists a relationship between prey density and the strategy adopted by predators. Aggressive behavior is connected to high prey and low predator densities, whereas a polymorphism dove-hawk is found at low prey and high predator densities. This result is consistent well with the previous studies of a domestic cat population [33, 35]. In rural areas, low density, most of the individuals are aggressive while in urban areas, high density, most of them are dove individuals.

In addition, we found that the equilibrium state of the system was determined by parameters $K, u, \alpha, a, C, r$. More 


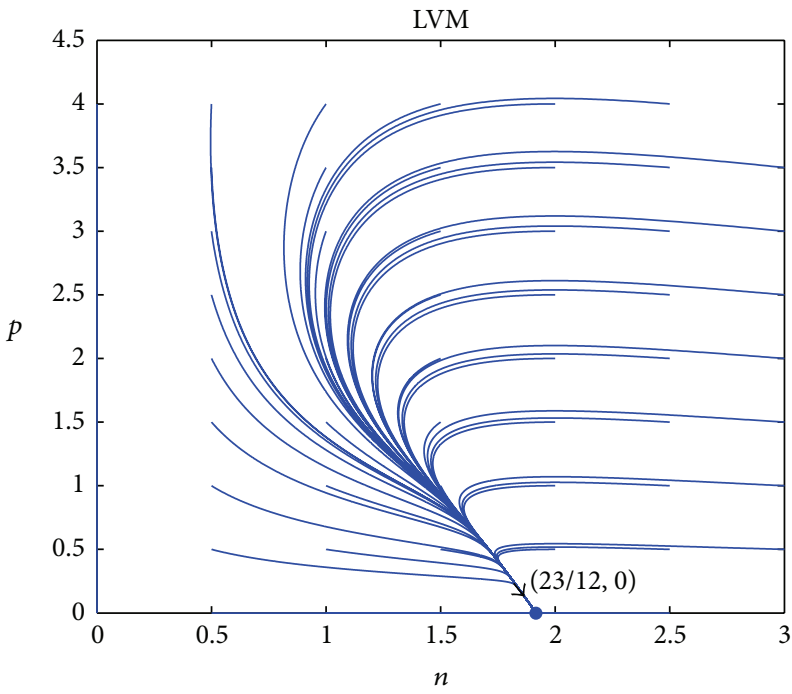

(a)

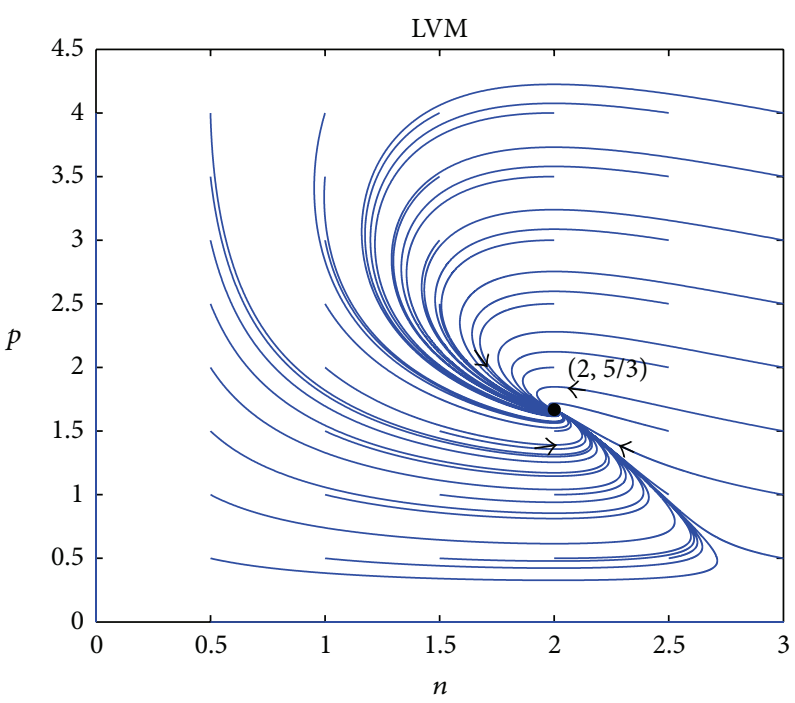

(c)

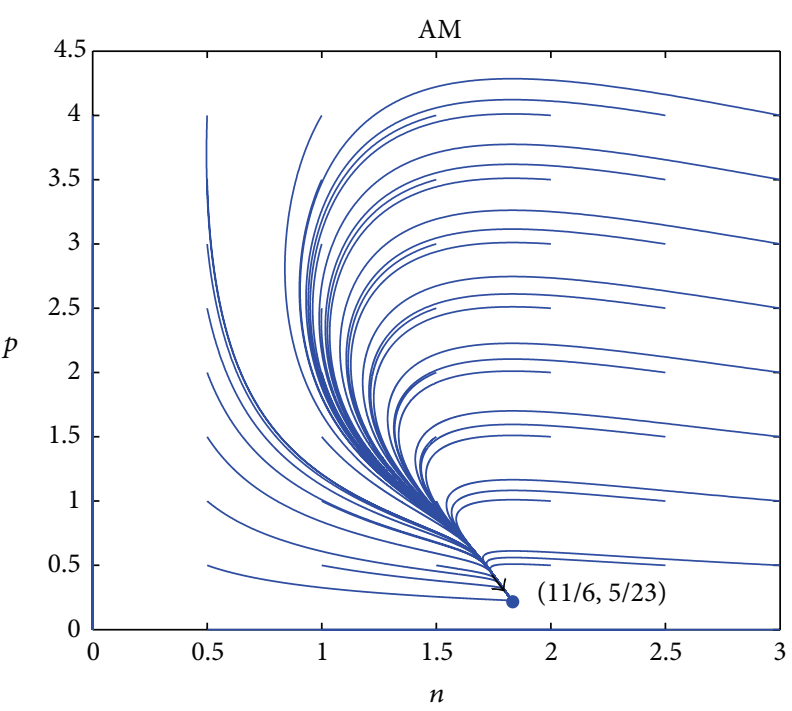

(b)

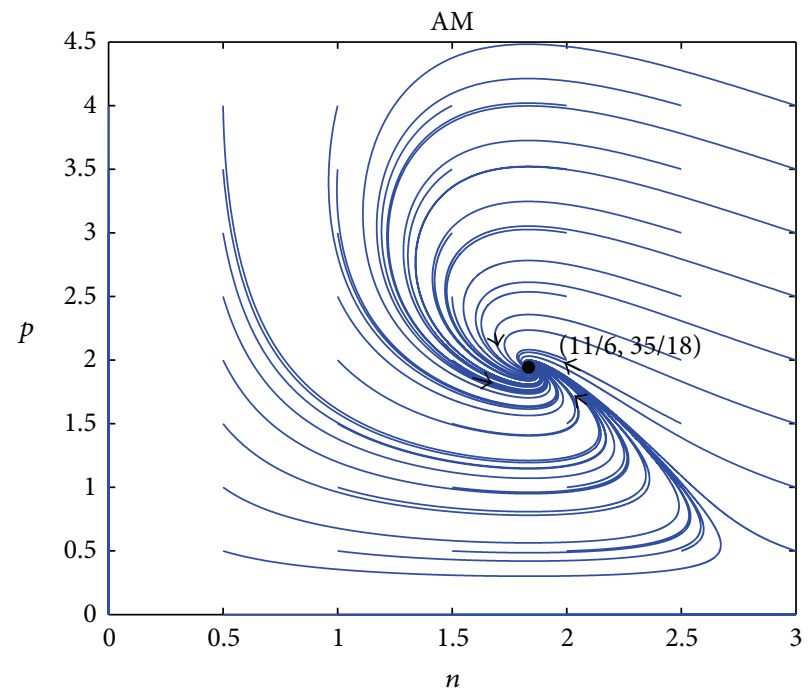

(d)

FIgURE 1: The dynamics of LVM and AM given the condition $C<u / \alpha$. Parameters are $r=5, K=23 / 12, a=1, \alpha=1, C=3 / 2$, and $u=2$ in the first two figures, the carrying capacity is set to be $K=3$ in the other two.

importantly, the change of the stable state by considering predator behavior with R-P-S strategy through comparing with the common Lotka-Volterra system without considering it can be observed, which also depends on $K, u, \alpha, a, C$, but not relates to $r$ (the birth rate of prey). Explicitly, it can be firstly recognized as two classes according to the numerical relationship of $C$ and $u / a$. Furthermore, in every class, constrained by the relationship of $K$ with $u / \alpha a$ and $(2 u+\alpha C) /(3 \alpha a)$, it appears that either a change from the extinction of predator populations in LVM to the coexistence state with predators and preys in AM, or from the coexistence in LVM to the extinction of predator population in AM, or coexistence state will be kept in both situations but with a change of the density of the population.
Especially, it is worth noting that given the conditions of $C<u / a$ and $K>u /(\alpha a)$, that is, when carrying capacity of predators is large enough and fighting cost of predators is small, coexistence state will be kept in both LotkaVolterra and Aggregated system, it shows an increase of the density of predator population and a decrease of the density of prey population. This verified the nature rule that an immediate competition will benefit the survival of the population. We also concluded that a diversification behavior of the predator population would maintain the stability of the ecosystem for certain parameters; that is, $C<u / a$ and $(2 u+\alpha C) /(3 \alpha a)<K<u /(\alpha a)$. Predator population will not go extinct by taking different strategies when disputing a prey. 


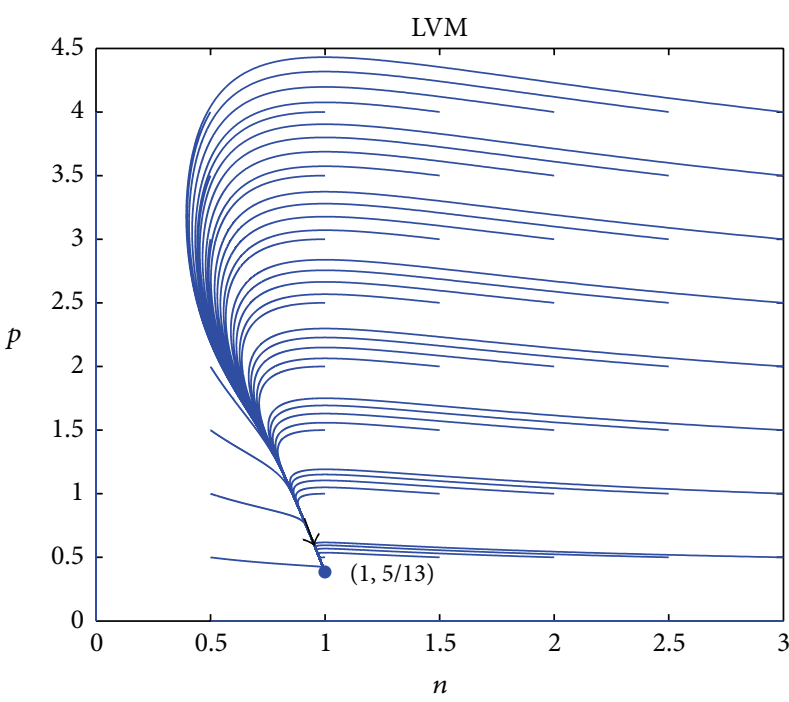

(a)

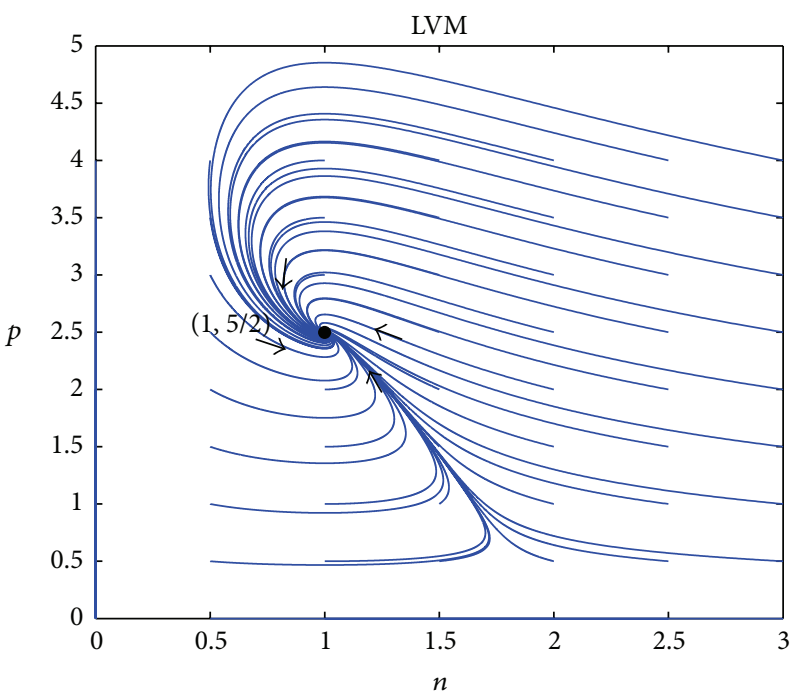

(c)

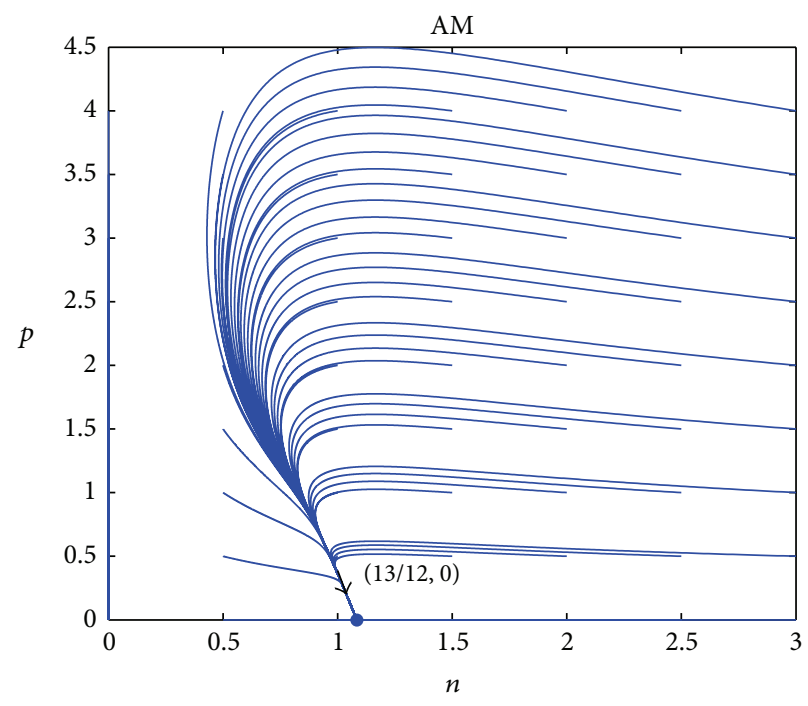

(b)

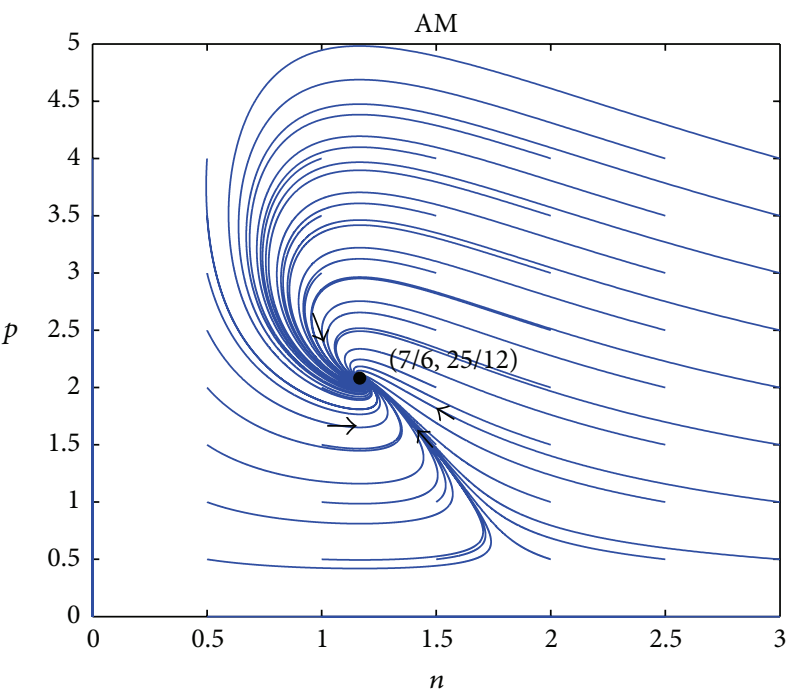

(d)

Figure 2: The dynamics of LVM and AM given the condition $C>u / \alpha$. Parameters are $r=5, K=13 / 12, a=1, \alpha=1, C=3 / 2$, and $u=2$ in the first two figures, all parameter values are the same in the other two except $K=2$.

In future, some more interesting researches are expected to expand by considering other intricate strategies taken by predators which would raise diverse ESS situations depending on different levels of prey densities, thus leading to diversification of the system. In addition, motivated by Huffaker's experiments [38], it has been documented that the distribution of prey and predators in the spatial configuration as well as the dispersal behaviors are all important factors to influence the dynamics of the predator-prey system; these factors play an important role in understanding the emergence of biodiversity and the stability of ecosystems (i.e., [39-42]). From this point of view, combining spatial effect into our model also deserves research, further studies are expected along this direction.

\section{Appendix}

\section{A. Local Stability of the Equilibria}

\section{A.1. Case of Model (14)}

The stability analysis of the aggregated model (14), the Jacobian matrix is

$$
\mathbf{J}=\left(\begin{array}{cc}
r\left(1-\frac{2 n}{K}\right)-a p & -a n \\
\frac{3 \alpha a p}{2} & -\mu+\frac{3 \alpha a n}{2}-\frac{\alpha C}{2}
\end{array}\right) .
$$


At the origin point $(0,0)$, we have

$$
\mathbf{J}_{(\mathbf{0 , 0 )}}=\left(\begin{array}{cc}
r & 0 \\
0 & -\mu-\frac{\alpha C}{2}
\end{array}\right) .
$$

It has two real eigenvalues with opposite signs, the origin point is a saddle point.

For the equilibrium point $(K, 0)$, the Jacobian matrix reads as follows:

$$
\mathbf{J}_{(\mathbf{K}, \mathbf{0})}=\left(\begin{array}{cc}
-r & -a K \\
0 & -\mu+\frac{3 \alpha a K}{2}-\frac{\alpha C}{2}
\end{array}\right),
$$

if $K<(2 u+\alpha C) / 3 \alpha a,(K, 0)$ is an asymptotically stable point, while for $K>(2 u+\alpha C) / 3 \alpha a$, a saddle point.

For the positive equilibrium point $(\bar{n}, \bar{p})=((2 u+$ $\left.\alpha C) /(3 \alpha a), r(3 \alpha a K-2 u-\alpha C) /\left(3 \alpha a^{2} K\right)\right)$, the Jacobian matrix reads as follows:

$$
\left.\mathbf{J}\right|_{(\overline{\mathbf{n}}, \overline{\mathbf{p}})}=\left(\begin{array}{cc}
-\frac{r}{K} \bar{n} & -a \bar{n} \\
\frac{3 \alpha a \bar{p}}{2} & 0
\end{array}\right) .
$$

So it is straightforward that when the initial state of the population belongs to the positive quadrant, the trace of the Jacobian matrix is negative and the determinant is positive which implies stability.

\section{A.2. Case of Model (15)}

The stability analysis of the Lotka-Volterra model (15): the Jacobian matrix is

$$
\mathbf{J}=\left(\begin{array}{cc}
r\left(1-\frac{2 n}{K}\right)-a p & -a n \\
\alpha a p & -\mu+\alpha a n
\end{array}\right) .
$$

At the origin point, we have

$$
\mathbf{J}_{(\mathbf{0}, \mathbf{0})}=\left(\begin{array}{cc}
r & 0 \\
0 & -\mu
\end{array}\right)
$$

with two real eigenvalues with opposite signs. The origin point is a saddle point.

For the equilibrium point $(K, 0)$, the Jacobian matrix reads as follows:

$$
\mathbf{J}_{(\mathbf{K}, \mathbf{0})}=\left(\begin{array}{cc}
-r & -a K \\
0 & -\mu+\alpha a K
\end{array}\right) .
$$

if $K>\mu /(\alpha a),(K, 0)$ is a saddle point, while for $K<\mu /(\alpha a)$, $(K, 0)$ is a stable node.

For the positive equilibrium point $\widetilde{x}=(\widetilde{n}, \tilde{p})=(u / \alpha a$, $(r / a)(1-u / \alpha a K))$, the Jacobian matrix reads as follows:

$$
\mathbf{J}_{(\tilde{\mathbf{n}}, \tilde{\mathbf{p}})}=\left(\begin{array}{cc}
-\frac{r}{K} \tilde{n} & -a \tilde{n} \\
\alpha a \tilde{p} & 0
\end{array}\right),
$$

where it is obvious that when the point belongs to the positive quadrant, the trace of the Jacobian matrix is negative; consequently, the determinant is positive so the equilibrium point is stable.

\section{A.3. Limit cycle analysis to system (14)}

We assume that there is a closed orbit $\Gamma_{0}$ around the interior point $\bar{x}$, the equation is

$$
n=n(t), \quad p=p(t), \quad-\infty<t<+\infty .
$$

Then there must be a $T>0$, where $n(T)=0, p(T)=0$. Next, we calculate the index $\gamma_{0}$ of $\Gamma_{0}$,

$$
\gamma_{0}=\frac{1}{T} \int_{0}^{T}\left[P_{n}^{\prime}(n(t), p(t))+Q_{p}^{\prime}(n(t), p(t))\right] d t
$$

where $P(n, p)=n(r-r(n / K)-a p)$ and $Q(n, p)=p(-\mu+$ $3 \alpha a n / 2-\alpha C / 2)$. Thus, (15) can be rewritten as

$$
\begin{gathered}
r-r \frac{n}{K}-a p=\frac{\mathrm{d} n}{n} \\
-\mu+\frac{3 \alpha a n}{2}-\frac{\alpha C}{2}=\frac{\mathrm{d} p}{p} .
\end{gathered}
$$

Calculating the equations on two sides, we can get

$$
\begin{gathered}
\frac{1}{T} \int_{0}^{T}\left[r-r \frac{n(t)}{K}-a p(t)\right] d t=\int_{n(0)}^{n(T)} \frac{\mathrm{d} n}{n}=\ln |n| \|_{n(0)}^{n(T)} \\
\frac{1}{T} \int_{0}^{T}\left[-\mu+\frac{3 \alpha a n(t)}{2}-\frac{\alpha C}{2}\right] d t=\int_{p(0)}^{p(T)} \frac{\mathrm{d} p}{p}=\ln \mid p \|_{p(0)}^{p(T)}
\end{gathered}
$$

It shows that $(1 / T) \int_{0}^{T} n(t) d t$ and $(1 / T) \int_{0}^{T} p(t) d t$ also satisfy the following algebra equation:

$$
\begin{gathered}
r-r \frac{n}{K}-a p=0 \\
-\mu+\frac{3 \alpha a n}{2}-\frac{\alpha C}{2}=0 .
\end{gathered}
$$


Therefore,

$$
\begin{aligned}
\frac{1}{T} \int_{0}^{T} n(t) d t & =\bar{n} \\
\frac{1}{T} \int_{0}^{T} p(t) d t & =\bar{p} .
\end{aligned}
$$

Then the index of $\Gamma_{0}$ is

$$
\begin{aligned}
\gamma_{0} & =\frac{1}{T} \int_{0}^{T}\left(r-2 r \frac{n}{K}-a p+-\mu+\frac{3 \alpha a n}{2}-\frac{\alpha C}{2}\right) d t \\
& =\frac{1}{T} \int_{0}^{T}\left(-r \frac{n}{K}\right) d t=-r \frac{\bar{n}}{K}<0 .
\end{aligned}
$$

Therefore, the closed orbit should be stable, it contradicts that $\overline{\mathrm{x}}$ is an asymptotically stable equilibrium, so the assumption is wrong; there are not any closed orbits.

\section{Conflict of Interests}

The authors declare that there is no conflict of interests regarding the publication of this paper.

\section{Acknowledgments}

Hui Zhang gratefully acknowledges the support from the National Natural Science Foundation of China (no. 31300310, no. 71171163, and no. 71271171), the Foundation Sciences Northwestern Polytechnical University (no. GDKY1007), the Projects of International Cooperation and Exchanges NSFC (no. 71311120091), and the Shaanxi Province Science and Technology Research and Development Programm (no. 2014KW03-01). Zhihui Ma gratefully acknowledges the support of the National Natural Science Foundation of China (no. 11301238).

\section{References}

[1] T. C. Gard, "Aggregation in stochastic ecosystem models," Ecological Modelling, vol. 44, no. 1-2, pp. 153-164, 1988.

[2] R. Bravo de la Parra, P. Auger, and E. Snchez, "Aggregation methods in discrete models," Journal of Biological Systems, vol. 3, pp. 603-612, 1995.

[3] Y. Iwasa, V. Andreasen, and S. Levin, "Aggregation in model ecosystems. I. Perfect aggregation," Ecological Modelling, vol. 37, no. 3-4, pp. 287-302, 1987.

[4] Y. Iwasa, S. A. Levin, and V. Andreasen, "Aggregation in model ecosystems. II. Approximate aggregation," IMA Journal of Mathematics Applied in Medicine and Biology, vol. 6, no. 1, pp. 1-23, 1989.

[5] P. Auger and R. Bravo de la Parra, "Methods of aggregation of variables in population dynamics," Comptes Rendus de l'Academie des Sciences, vol. 323, no. 8, pp. 665-674, 2000.

[6] P. Auger, R. Bravo de la Parra, S. Morand, and E. Sánchez, "A predator-prey model with predators using hawk and dove tactics," Mathematical Biosciences, vol. 177-178, pp. 185-200, 2002.

[7] J. M. Smith and G. R. Price, "The logic of animal conflict," Nature, vol. 246, no. 5427, pp. 15-18, 1973.
[8] A. J. Lotka, "Undamped oscillations derived from the law of mass action," Journal of the American Chemical Society, vol. 42, no. 8, pp. 1595-1598, 1920.

[9] V. Volterra, "Variation e fluttuazioni del numero d'individui in specie animali conviventi," Memoria della Regia Accademia Nazionale dei Lincei, vol. 2, pp. 31-113, 1926.

[10] C. Cattani and A. Ciancio, "Qualitative analysis of second-order models of tumor-immune system competition," Mathematical and Computer Modelling, vol. 47, no. 11-12, pp. 1339-1355, 2008.

[11] C. Cattani and A. Ciancio, "A class of solutions for the hybrid kinetic model in the tumor-immune system competition," Mathematical Problems in Engineering, vol. 2013, Article ID 430486, 11 pages, 2013.

[12] C. Cattani and A. Ciancio, "Separable transition density in the hybrid model for tumor-immune system competition," Computational and Mathematical Methods in Medicine, vol. 2012, Article ID 610124, 6 pages, 2012.

[13] R. Colucci, "Coexistence in a one-predator, two-prey system with indirect effects," Journal of Applied Mathematics, vol. 2013, Article ID 625391, 13 pages, 2013.

[14] P. Chesson, "Mechanisms of maintenance of species diversity," Annual Review of Ecology and Systematics, vol. 31, pp. 343-366, 2000.

[15] Å. Brännström, N. Loeuille, M. Loreau, and U. Dieckmann, "Emergence and maintenance of biodiversity in an evolutionary food-web model," Theoretical Ecology, vol. 4, no. 4, pp. 467-478, 2011.

[16] J. M. Levine and J. HilleRisLambers, "The maintenance of species diversity," Nature Education Knowledge, vol. 3, article 59, 2012.

[17] J. M. Biernaskie, A. Gardner, and S. A. West, "Multicoloured greenbeards, bacteriocin diversity and the rock-paper-scissors game," Journal of Evolutionary Biology, vol. 26, pp. 2081-2094, 2013.

[18] R. M. Sibly and R. H. Smith, Eds., Behavioural Ecology: Ecological Consequences of Adaptive Behaviours, Blackwell, Oxford, UK, 1985.

[19] A. Lømnicki, "Regulation of population density due to individual differences and patchy environment," Oikos, vol. 35, pp. 185193, 1988.

[20] M. Pigliucci, Phenotypic Plasticity: Beyond Nature and Nurture, Johns Hopkins University Press, Baltimore, Md, USA, 2001.

[21] V. Grimm and S. F. Railsback, Individual-Based Modeling and Ecology, Princeton University Press, Princeton, NJ, USA, 2005.

[22] J. A. Stamps and M. Beuchner, "The territorial defense hypothesis and the ecology of insular vertebrates," Quarterly Review of Biology, vol. 60, no. 2, pp. 155-181, 1985.

[23] P. Perret and J. Blondel, "Experimental evidence of the territorial defense hypothesis in insular Blue Tits," Experientia, vol. 49, no. 1, pp. 94-98, 1993.

[24] D. Pontier, N. Rioux, and A. Heizmann, "Evidence of selection on the orange allele in the domestic cat Felis catus: the role of social structure," Oikos, vol. 73, no. 3, pp. 299-308, 1995.

[25] P. D. Taylor and L. B. Jonker, "Evolutionarily stable strategies and game dynamics," Mathematical Biosciences, vol. 40, no. 1-2, pp. 145-156, 1978.

[26] J. Hofbauer, P. Schuster, and K. Sigmund, "A note on evolutionary stable strategies and game dynamics," Journal of Theoretical Biology, vol. 81, no. 3, pp. 609-612, 1979.

[27] E. C. Zeeman, "Population dynamics from game theory," in Proceedings of an International Conference on Global Theory of 
Dynamical Systems, A. Nitecki and C. Robinson, Eds., vol. 819 of Lecture Notes in Mathematics, Springer, Berlin, Germany.

[28] J. W. Weibull, Evolutionary Game Theory, MIT Press, Cambridge, Mass, USA, 1995.

[29] J. Hofbauer and K. Sigmund, Evolutionary Games and Population Dynamics, Cambridge University Press, Cambridge, UK, 1998.

[30] J. Hofbauer and K. Sigmund, "Evolutionary game dynamics," Bulletin of the American Mathematical Society, vol. 40, no. 4, pp. 479-519, 2003.

[31] W. S. C. Gurney and R. M. Nisbet, Ecological Dynamics, Oxford University, Oxford, UK, 1998.

[32] E. Sanchez, P. Auger, and R. Bravo de la Parra, "Influence of individual aggressiveness on the dynamics of competitive populations," Acta Biotheoretica, vol. 45, no. 3-4, pp. 321-333, 1997.

[33] P. Auger and D. Pontier, "Fast game theory coupled to slow population dynamics: the case of domestic cat populations," Mathematical Biosciences, vol. 148, no. 1, pp. 65-82, 1998.

[34] P. Auger, R. Bravo de la Parra, and E. Sánchez, "Hawk-dove game and competition dynamics," Mathematical and Computer Modelling, vol. 27, no. 4, pp. 89-98, 1998.

[35] D. Pontier, P. Auger, R. Bravo de la Parra, and E. Sánchez, “The impact of behavioral plasticity at individual level on domestic cat population dynamics," Ecological Modelling, vol. 133, no. 1-2, pp. 117-124, 2000.

[36] M. M. Peixoto, “On structural stability," Annals of Mathematics. Second Series, vol. 69, pp. 199-222, 1959.

[37] M. Mangel and C. W. Clark, Dynamic Modeling in Behavioral Ecology, Princeton University Press, Princeton, NJ, USA, 1988.

[38] C. B. Huffaker, "Experimental studies on predation: dispersion factors and predatorprey oscillations," Hilgardia, vol. 27, pp. 343-383, 1958.

[39] M. Mobilia, I. T. Georgiev, and U. C. Täuber, "Fluctuations and correlations in lattice models for predator-prey interaction," Physical Review E, vol. 73, no. 4, Article ID 040903, 2006.

[40] Q. He, U. C. Täuber, and R. K. P. Zia, "On the relationship between cyclic and hierarchical three-species predator-prey systems and the two-species Lotka-Volterra model," The European Physical Journal B, vol. 85, no. 4, pp. 141-153, 2012.

[41] K. J. Benoit-Bird, B. C. Battaile, S. A. Heppell et al., "Prey patch patterns predict habitat use by top marine predators with diverse foraging strategies," PLOS ONE, vol. 8, no. 1, Article ID e53348, 2013.

[42] M. Fras and M. Gosak, "Spatiotemporal patterns provoked by environmental variability in a predator-prey model," Biosystems, vol. 114, pp. 172-177, 2013. 


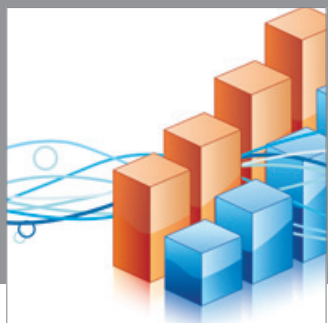

Advances in

Operations Research

mansans

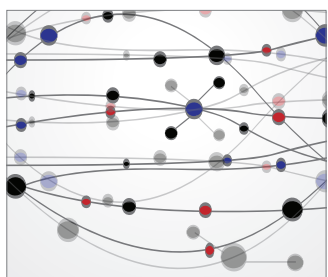

The Scientific World Journal
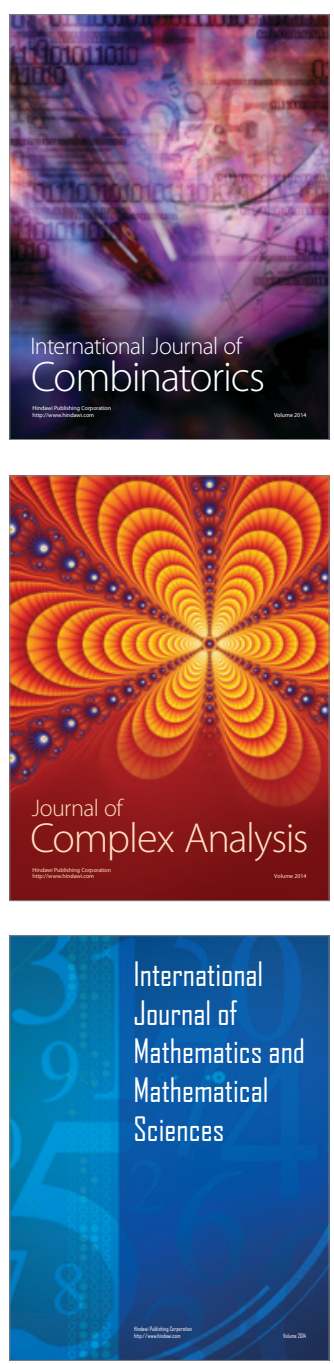
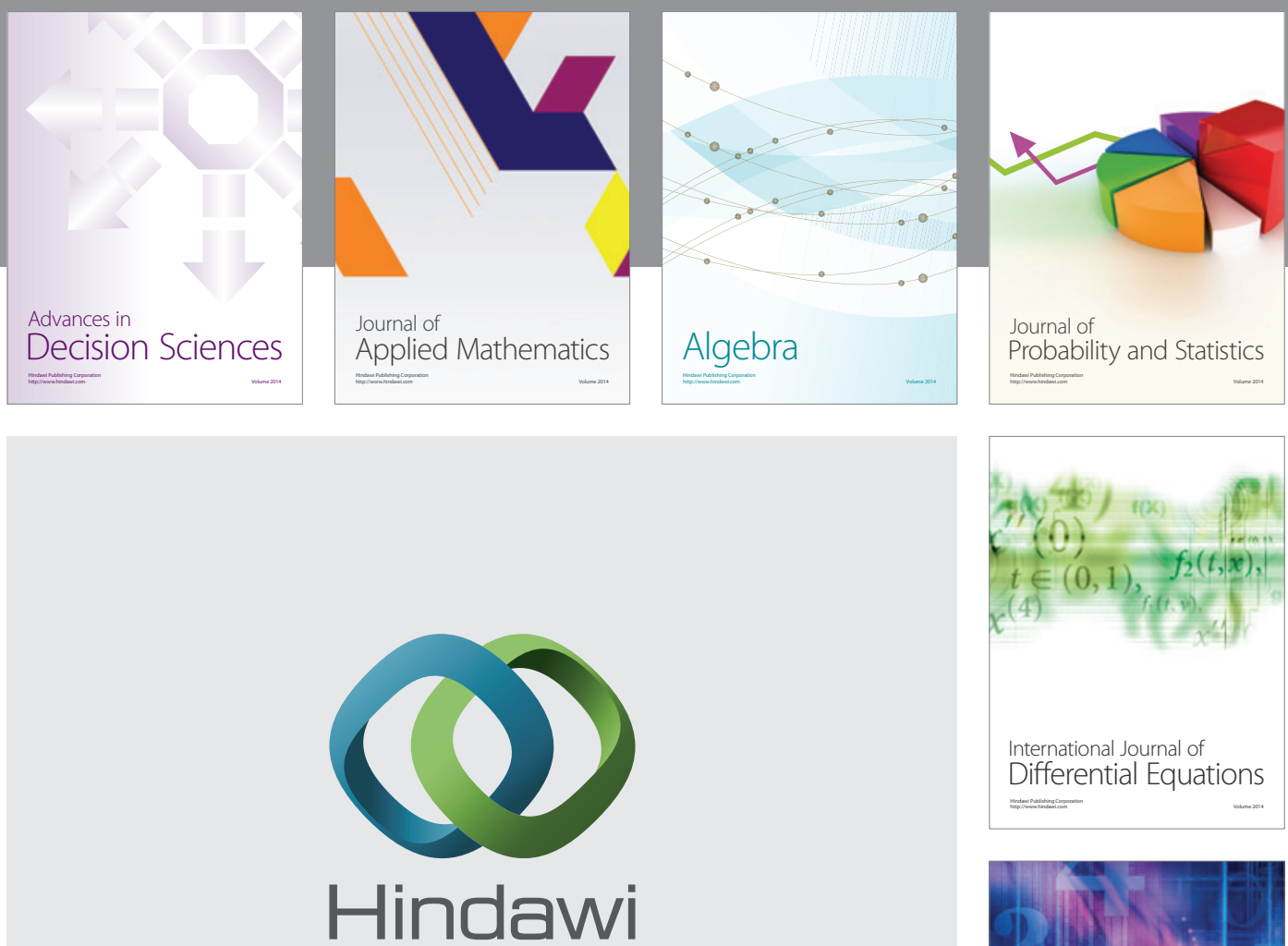

Submit your manuscripts at http://www.hindawi.com
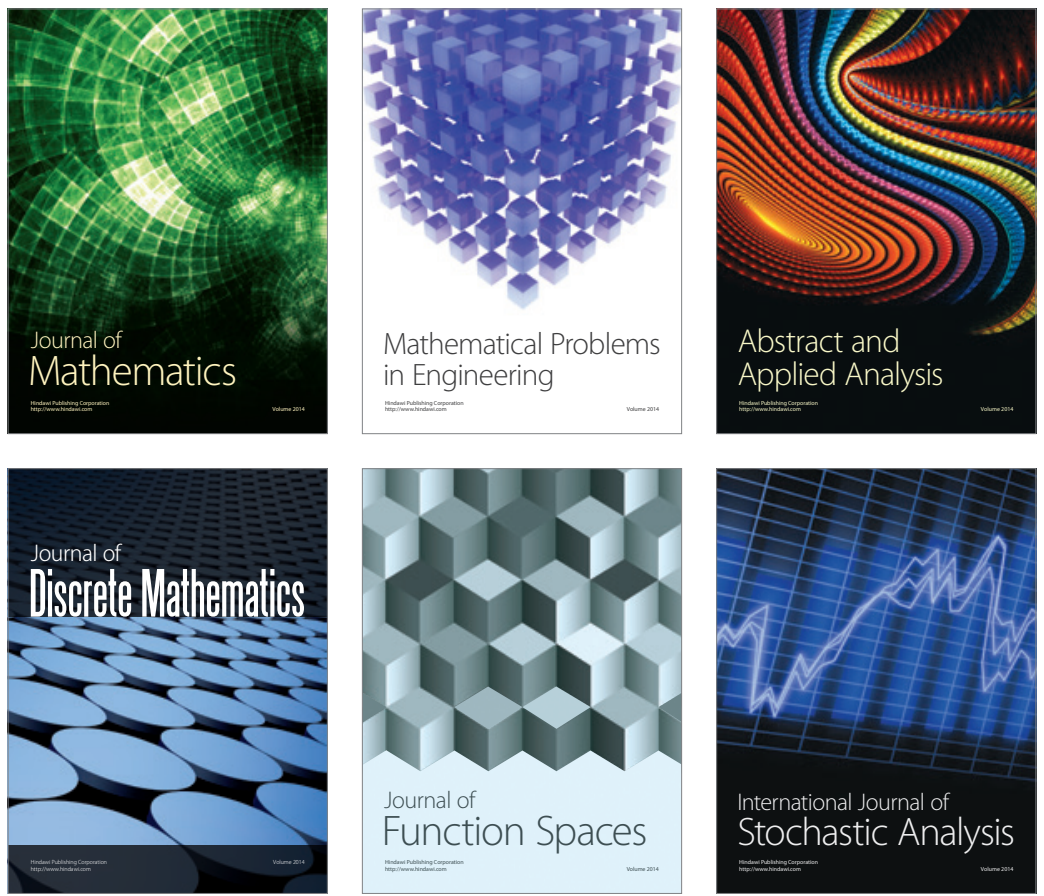

Journal of

Function Spaces

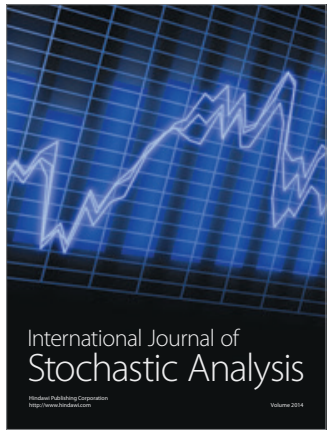

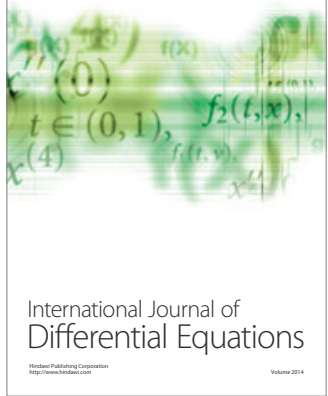
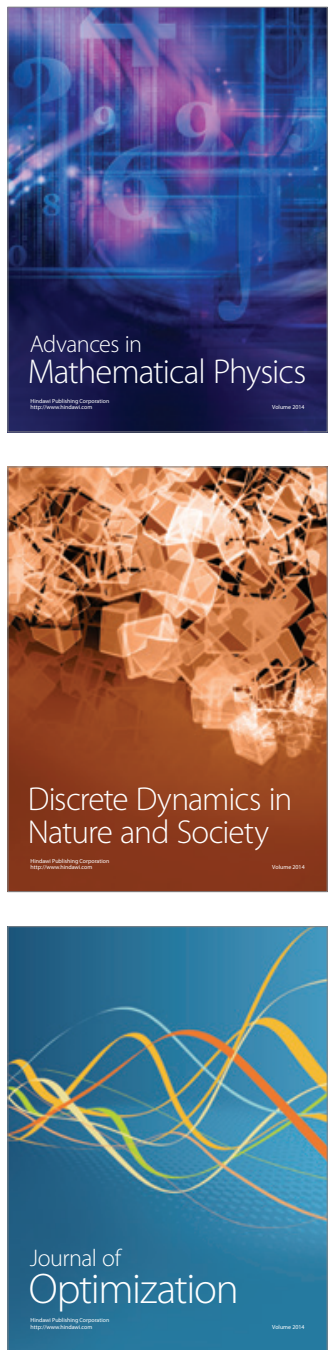